\title{
Comparação do perfil nutricional, lipídico e glicêmico de crianças e adolescentes de diferentes hemisférios da zona rural de Santa Cruz do Sul - RS
}

\author{
Comparison of the nutritional, lipidic and glycemic profile of children \\ and adolescents of different hemispheres of the rural area of Santa \\ cruz do Sul - RS
}

\begin{abstract}
Cláudia Daniela Barbian, ${ }^{1}$ Leandro Tibiriçá Burgos, ${ }^{1}$ Letícia Welser, ${ }^{1}$ Ana Paula Sehn, ${ }^{1}$ Deise Graziela Kern, ${ }^{1}$ Cristiane Fernanda da Silva, ${ }^{1}$ Cézane Priscila Reuter ${ }^{1}$ 'Universidade de Santa Cruz do Sul (UNISC), Santa Cruz do Sul, RS, Brasil.
\end{abstract}

Recebido em: 16/02/2017 / Aceito em: 09/03/2017 / Publicado em: 31/03/2017 claubarbian@hotmail.com

\section{RESUMO}

Fatores de risco para doenças cardiovasculares estão presentes na obesidade infanto-juvenil, como a dislipidemia e a hiperinsulinemia. Objetivo: verificar o perfil nutricional, glicêmico e lipídico em escolares, comparando os hemisférios norte, sul, leste e oeste da zona rural de Santa Cruz do Sul - RS. Método: estudo de caráter transversal, com 729 escolares da zona rural, com idades entre sete e 15 anos. Duas escolas apresentaram características rurais e três escolas características urbanas. Foi realizada coleta sanguínea para verificação do colesterol total (CT), colesterol HDL (HDL-c), colesterol LDL (LDL-c), triglicerídeos (TG) e glicose. Foi realizada a mensuração do peso e estatura para cálculo do índice de massa corporal. Para a análise estatística, foi utilizado o programa SPSS 20.0 e a comparação das variáveis categóricas por escola foi realizada por meio do teste qui-quadrado, sendo o valor de $p<0,05$ considerado significante. Resultados: $27 \%$ dos escolares apresentaram sobrepeso e obesidade, 59,7\%, 52,4\%, $21,8 \%$ e $30,5 \%$ apresentaram LDL-c, CT, TG e glicose alterados, respectivamente, e $21 \%$ HDL-c diminuídos. Quanto ao comparativo entre escolas, houve diferença significativa entre as escolas em todas as variáveis bioquímicas para ambos os sexos. Considerações finais: os escolares apresentaram expressivas alterações bioquímicas, principalmente nas escolas com características urbanas, resultado este que pode vir a colaborar para futuros problemas cardiovasculares.
Descritores: Lipídeos; Glicemia; Criança; Adolescente; Saúde Escolar.

\section{ABSTRACT}

Risk factors for cardiovascular diseases are present in childhood and juvenile obesity, such as dyslipidemia and hyperinsulinemia. Objective: to verify the nutritional, glycemic and lipid profile in schoolchildren, comparing the northern, southern, eastern and western hemispheres of the rural area of Santa Cruz do Sul - RS. Method: cross-sectional study, with 729 rural schoolchildren, aged between 7 and 15 years. Two schools had rural characteristics and three urban schools. Blood samples were collected for total cholesterol, HDL-cholesterol, LDL-cholesterol, triglycerides (TG), and glucose. Weight and height were measured to calculate the body mass index. Statistical analysis, was performed using the SPSS 20.0 program, and the comparison of the categorical variables by school was performed using the chi-square test, with $p<0.05$ considered significant. Results: $27 \%$ of the students were overweight and obese, 59.7\%, 52.4\%, 21.8\% and $30.5 \%$ presented altered LDL-C, CT, TG and glucose, respectively, and $21 \%$ HDL-c Decreased. Regarding the comparison between schools, there was a significant difference between schools in all biochemical variables for both sexes. Closing remarks: schoolchildren presented significant biochemical changes, especially in 
schools with urban characteristics, a result that may contribute to future cardiovascular problems.

Keywords: Lipids; Glycemia; Child; Adolescent; School Health.

\section{INTRODUÇÃO}

É evidente, nos dias atuais, a proporção que a obesidade atingiu no mundo todo, sendo a mesma percebida em diferentes idades, raças e em ambos os sexos. ${ }^{1}$ A obesidade, condição crônica caracterizada pelo acúmulo de gordura corporal, torna-se coadjuvante no surgimento de múltiplas doenças, acarretando em riscos à saúde. ${ }^{2}$

O aumento da prevalência de sobrepeso e obesidade infanto-juvenil evidencia, ainda mais, os fatores de risco ligados a esse distúrbio metabólico, ${ }^{3}$ como o diabetes mellitus, ${ }^{4}$ a intolerância à glicose, a dislipidemia e outras comorbidades. ${ }^{5}$ Essas alterações facilitam o desenvolvimento de síndrome metabólica, bem como auxiliam a elevação dos valores de triglicerídeos e redução das taxas de colesterol HDL (HDL-c; high density lipoprotein). ${ }^{6}$

Outra preocupação, juntamente a essas alterações, é o fato de indivíduos acometidos por excesso de peso na infância, tornam-se predispostos a desenvolver na vida adulta, obesidade e outros problemas de saúde, como doenças cardiovasculares e síndrome metabólica. ${ }^{7}$ Concomitantemente, Sobrero et al. ${ }^{8}$ citam que a síndrome metabólica e o risco cardiometabólico são determinados pela obesidade, sobretudo a gordura central. Do mesmo modo, a obesidade abdominal facilita o surgimento de fatores de risco metabólicos e cardiovasculares. ${ }^{9}$ Bergmann et al. ${ }^{10}$ relatam que o excesso de peso está vinculado a variáveis que predispõem a doenças cardiovasculares, sendo estas uma das maiores causas de mortalidade no mundo. Fatores de risco para doenças cardiovasculares também estão presentes na obesidade infanto-juvenil, como a dislipidemia e a hiperinsulinemia. ${ }^{11}$

Níveis alterados no perfil lipídico, como colesterol total (CT), colesterol LDL (LDL-c), HDL-c e triglicerídeos estão relacionados com a hipertensão arterial e doenças ateroscleróticas, ${ }^{12}$ bem como a dislipidemia associa-se positivamente ao excesso de peso e a hábitos alimentares. ${ }^{13}$ Com o aumento dos casos de risco para doenças cardiovasculares, se faz necessário a investigação de quadros alterados no perfil lipídico na faixa etária infanto-juvenil, tendo em vista que o excesso de peso influencia nos níveis das variáveis que compõe esse perfil. ${ }^{14}$

Neste contexto, o objetivo do presente estudo é verificar o perfil nutricional, glicêmico e lipídico de escolares de sete a 15 anos, comparando os hemisférios norte, sul, leste e oeste, da zona rural de Santa Cruz do Sul - RS.

\section{MÉTODO}

Foi realizado um estudo transversal, com uma amostra de 729 escolares com idades entre sete e 15 anos, sendo 352 do sexo masculino e 377 do sexo feminino, alunos de cinco escolas da zona rural do município de Santa Cruz do Sul - RS (Quadro 1). O presente estudo faz parte de uma pesquisa desenvolvida na Universidade de Santa Cruz do Sul (UNISC), intitulada "Avaliação de indicadores bioquímicos de saúde de escolares usando espectroscopia no infravermelho, polimorfismos, saúde bucal e fatores relacionados ao estilo de vida: um estudo em Santa Cruz do Sul - Fase II", já aprovada pelo Comitê de Ética em Pesquisa com Seres Humanos da UNISC, sob protocolo número 3044/11.

A coleta dos dados, primeiramente, constou de um contato com cada uma das direções das cinco escolas da rede pública participantes, visando demonstrar a importância da investigação dos fatores de risco associados à obesidade. Obtida a concordância, foi enviado um questionário com questões referentes a estilo de vida, saúde e bem-estar, assim como o termo de consentimento livre e esclarecido aos pais e/ou responsáveis pela criança ou adolescente. Obtida a autorização, os escolares foram convidados a comparecer à UNISC, em dias pré-estabelecidos na parte da manhã, após um jejum prévio de no mínimo $12 \mathrm{~h}$, para a coleta de sangue para a realização dos exames laboratoriais (colesterol total, HDL-c, LDL-c e triglicerídeos). Após, foram realizados demais procedimentos como mensuração do peso e estatura com auxílio de uma balança antropométrica devidamente calibrada.

O perfil lipídico foi avaliado de acordo com os valores do National Heart, Lung, and Blood Institute. ${ }^{15}$ Foram considerados alterados os resultados nas classes limítrofe e aumentado, para colesterol total $(\geq 170$ $\mathrm{mg} / \mathrm{dL}), \mathrm{LDL}-\mathrm{c}(\geq 110 \mathrm{mg} / \mathrm{dL}$ ) e triglicerídeos ( $\geq 75 \mathrm{mg} /$ $\mathrm{dL}$ para crianças e $\geq 90 \mathrm{mg} / \mathrm{dL}$ para adolescentes) e limítrofe e baixo para HDL-c $(\leq 45 \mathrm{mg} / \mathrm{dL})$. Para a glicose, foi utilizado o protocolo do American Diabetes Association, ${ }^{16}$ sendo considerado alterado os escolares com pré-diabetes (100-126 mg/dL) e diabetes $(\geq 126$ $\mathrm{mg} / \mathrm{dL}$ ). A classificação do IMC foi realizada de acordo com sexo e idade, considerando baixo peso $(<p 5)$, normal $(\geq p 5$ e $<p 85)$, sobrepeso $(p \geq 85$ e $<p 95)$ e obesidade ( $\geq \mathrm{p} 95$ ), conforme as curvas e percentis do Centers for Disease Control and Prevention/National Center for Health Statistics. ${ }^{17}$

As análises estatísticas realizadas no programa SPSS (Statistical Package for the Social Sciences) versão 20.0, através da análise descritiva (frequência e percentual). Para comparação das variáveis categóricas por escola foi utilizado o teste do qui-quadrado, sendo $o$ valor de $p<0,05$ considerado significante.

Quadro 1 - Localização e características das escolas.

\begin{tabular}{|l|c|c|l|c|c|}
\hline Escola & Hemisfério & $\begin{array}{c}\text { Distância do centro } \\
\text { da cidade }\end{array}$ & Características & Masc. & Fem. \\
\hline 1 & Sul & Aprox. $15 \mathrm{~km}$ & $\begin{array}{l}\text { Escola situada em região com muitas famílias de agricultores, que preservam } \\
\text { tradições saudáveis e os filhos ajudam na lavoura. }\end{array}$ & 50 & 50 \\
\hline 2 & Norte ${ }^{1}$ & Aprox. $30 \mathrm{~km}$ & Escola situada em uma vila com características urbanas. & 89 & 89 \\
\hline 3 & Leste & Aprox. $10 \mathrm{~km}$ & $\begin{array}{l}\text { Escola próxima a uma rodovia federal de grande fluxo, próximo ao centro da } \\
\text { cidade, e com várias linhas de ônibus. }\end{array}$ & 41 & 41 \\
\hline 4 & Oeste & Aprox. $20 \mathrm{~km}$ & Escola em região asfaltada, com comércio e linhas de ônibus em suas proximidades. & 108 & 108 \\
\hline 5 & Norte & Aprox. $50 \mathrm{~km}$ & $\begin{array}{l}\text { Escola distante da área urbana, com muitos aspectos rurais, e com filhos de } \\
\text { agricultores auxiliando na lavoura. }\end{array}$ & 64 & 64 \\
\hline
\end{tabular}




\section{RESULTADOS}

Das 729 crianças e adolescentes participantes, $51,7 \%$ eram do sexo feminino. A distribuição dos sujeitos por escola foi de: $113(15,5 \%)$ na Escola 1 (sul), $193(26,5 \%)$ na Escola 2 (norte $\left.{ }^{1}\right), 82(11,2 \%)$ na Escola 3 (leste), $216(29,7 \%)$ na Escola 4 (oeste) e $125(17,1 \%)$ na Escola 5 (norte $\left.{ }^{2}\right)$. O perfil de risco cardiovascular (nutricional, lipídico e glicêmico) dos escolares pode ser observado na tabela 1, em que se verificou no total da amostra, elevado índice de alteração nos fatores de risco avaliados, com destaque para o LDL-c $(59,7 \%)$, colesterol total $(52,4 \%)$ e glicose $(30,5 \%)$. Quanto ao perfil nutricional, $27,0 \%$ da amostra apresentou sobrepeso e obesidade. Quando estratificado por sexo, observou-se que, quanto as variáveis do perfil lipídico, bem como o IMC, no sexo feminino houve maiores percentuais de alterações, com maiores diferenças em relação aos valores de triglicerídeos, colesterol total e LDL-c. No sexo masculino ocorreu maior percentual de alteração na glicose, sendo $36,1 \%$ comparado a $25,2 \%$ do sexo feminino.

Os indicadores de saúde dos escolares do sexo masculino são apresentados na tabela 2. Houve diferença significativa $(p<0,001)$ em todas as variáveis avaliadas do perfil lipídico e na glicose, no comparativo entre as cinco escolas avaliadas. As escolas $4 \mathrm{e}$ 2 apresentaram, respectivamente, maiores percentuais de escolares com colesterol total alterado $161,1 \%$ e $52,8 \%)$, LDL-c alterado $(67,6 \%$ e $60,7 \%)$, glicose elevada $(53,7 \%$ e $49,4 \%)$ e triglicerídeos elevados $(20,4 \%$ e $25,8 \%$ ), bem como as escolas 1 e 4 apresentaram os maiores índices de sobrepeso e obesidade $(26,0 \%$ e $35,2 \%$, respectivamente). O HDL-c limítrofe obteve maior predomínio nas escolas $3(39,0 \%)$ e $5(25,0 \%)$.

A tabela 3 apresenta os indicadores de saúde de escolares do sexo feminino. As variáveis avaliadas do perfil lipídico e a glicose, no comparativo entre as cinco escolas avaliadas, foram estatisticamente significativas.

Tabela 1 - Indicadores de saúde dos sujeitos.

\begin{tabular}{|c|c|c|c|}
\hline & Total n (\%) & Feminino $\mathrm{n}(\%)$ & Masculino n (\%) \\
\hline \multicolumn{4}{|l|}{ IMC } \\
\hline Baixo peso/normal & $532(73,0)$ & $273(72,4)$ & $259(73,6)$ \\
\hline Sobrepeso/obesidade & $197(27,0)$ & $104(27,6)$ & $93(26,4)$ \\
\hline \multicolumn{4}{|l|}{ Triglicerídeos } \\
\hline Aceitável & $570(78,2)$ & $276(73,2)$ & $294(83,5)$ \\
\hline Limítrofe + aumentado & $159(21,8)$ & $101(26,8)$ & $58(16,6)$ \\
\hline \multicolumn{4}{|l|}{ Colesterol Total } \\
\hline Aceitável & $347(47,6)$ & $165(43,8)$ & $182(51,7)$ \\
\hline Limítrofe + aumentado & $382(52,4)$ & $212(56,2)$ & $170(48,3)$ \\
\hline \multicolumn{4}{|l|}{ Colesterol HDL-c } \\
\hline Aceitável & $576(79,0)$ & $296(78,5)$ & $280(79,5)$ \\
\hline Limítrofe + baixo & $153(21,0)$ & $81(21,5)$ & $72(20,5)$ \\
\hline \multicolumn{4}{|l|}{ LDL-c } \\
\hline Aceitável & $294(40,3)$ & $136(36,1)$ & $158(44,9)$ \\
\hline Limítrofe + aumentado & $435(59,7)$ & $241(63,9)$ & $194(55,1)$ \\
\hline \multicolumn{4}{|l|}{ Glicose } \\
\hline Normal & $507(69,5)$ & $282(74,8)$ & $225(63,9)$ \\
\hline Alterada & $222(30,5)$ & $95(25,2)$ & $127(36,1)$ \\
\hline Total da amostra & $729(100)$ & $377(51,7)$ & $352(48,3)$ \\
\hline
\end{tabular}

Tabela 2 - Indicadores de saúde de escolares do sexo masculino.

\begin{tabular}{|c|c|c|c|c|c|c|}
\hline & $\begin{array}{c}\text { Escola } 1 \text { Sul } \\
n(\%)\end{array}$ & $\begin{array}{c}\text { Escola } 2 \text { Norte }{ }^{1} \\
\text { n (\%) }\end{array}$ & $\begin{array}{c}\text { Escola } 3 \text { Leste } \\
\text { n (\%) }\end{array}$ & $\begin{array}{c}\text { Escola } 4 \text { Oeste } \\
\text { n (\%) }\end{array}$ & $\begin{array}{c}\text { Escola } 5 \text { Nordeste }^{2} \\
n(\%)\end{array}$ & $p$ \\
\hline \multicolumn{7}{|l|}{ IMC } \\
\hline Baixo peso/normal & $37(74,0)$ & $69(77,5)$ & $32(78,0)$ & $70(64,8)$ & $51(79,7)$ & 0,156 \\
\hline Sobrepeso/obesidade & $13(26,0)$ & $20(22,5)$ & $9(22,0)$ & $38(35,2)$ & $13(20,3)$ & \\
\hline \multicolumn{7}{|l|}{ Triglicerídeos } \\
\hline Aceitável & $41(82,0)$ & $66(74,2)$ & $40(97,6)$ & $86(79,6)$ & $61(95,3)$ & $<0,001$ \\
\hline Limítrofe + aumentado & $9(18,0)$ & $23(25,8)$ & $1(2,4)$ & $22(20,4)$ & $3(4,7)$ & \\
\hline \multicolumn{7}{|l|}{ Colesterol Total } \\
\hline Aceitável & $29(58,0)$ & $42(47,2)$ & $30(73,2)$ & $42(38,9)$ & $39(60,9)$ & $<0,001$ \\
\hline Limítrofe + aumentado & $21(42,0)$ & $47(52,8)$ & $11(26,8)$ & $66(61,1)$ & $25(39,1)$ & \\
\hline \multicolumn{7}{|l|}{ Colesterol HDL-c } \\
\hline Aceitável & $48(96,0)$ & $69(77,5)$ & $25(61,0)$ & $90(83,3)$ & $48(75,0)$ & $<0,001$ \\
\hline Limítrofe + baixo & $2(4,0)$ & $20(22,5)$ & $16(39,0)$ & $18(16,7)$ & $16(25,0)$ & \\
\hline \multicolumn{7}{|l|}{ LDL-c } \\
\hline Aceitável & $25(50,0)$ & $35(39,3)$ & $32(78,0)$ & $35(32,4)$ & $31(48,4)$ & $<0,001$ \\
\hline Limítrofe + aumentado & $25(50,0)$ & $54(60,7)$ & $9(22,0)$ & $73(67,6)$ & $33(51,6)$ & \\
\hline \multicolumn{7}{|l|}{ Glicose } \\
\hline Normal & $29(58,0)$ & $45(50,6)$ & $41(100,0)$ & $50(46,3)$ & $60(93,8)$ & $<0,001$ \\
\hline Alterada & $21(42,0)$ & $44(49,4)$ & $0(0,0)$ & $58(53,7)$ & $4(6,2)$ & \\
\hline
\end{tabular}


Tabela 3 - Indicadores de saúde de escolares do sexo feminino,

\begin{tabular}{|c|c|c|c|c|c|c|}
\hline & $\begin{array}{c}\text { Escola } 1 \text { Sul } \\
n(\%)\end{array}$ & $\begin{array}{c}\text { Escola } 2 \text { Norte }^{1} \\
\text { n (\%) }\end{array}$ & $\begin{array}{c}\text { Escola } 3 \text { Leste } \\
n(\%)\end{array}$ & $\begin{array}{c}\text { Escola } 4 \text { Oeste } \\
n(\%)\end{array}$ & $\begin{array}{c}\text { Escola } 5 \text { Nordeste }^{2} \\
n(\%)\end{array}$ & $p$ \\
\hline \multicolumn{7}{|l|}{ IMC } \\
\hline Baixo peso/normal & $49(77,8)$ & $74(71,2)$ & $31(75,6)$ & $78(72,2)$ & $41(67,2)$ & 0,731 \\
\hline Sobrepeso/obesidade & $14(22,2)$ & $30(28,8)$ & $10(24,4)$ & $30(27,8)$ & $20(32,8)$ & \\
\hline \multicolumn{7}{|l|}{ Triglicerídeos } \\
\hline Aceitável & $47(74,6)$ & $69(66,3)$ & $39(95,1)$ & $83(76,9)$ & $38(62,3)$ & 0,002 \\
\hline Limítrofe + aumentado & $16(25,4)$ & $35(33,7)$ & $2(4,9)$ & $25(23,1)$ & $23(37,7)$ & \\
\hline \multicolumn{7}{|l|}{ Colesterol Total } \\
\hline Aceitável & $24(38,1)$ & $33(31,7)$ & $33(80,5)$ & $45(41,7)$ & $30(49,2)$ & $<0,001$ \\
\hline Limítrofe + aumentado & $39(61,9)$ & $71(68,3)$ & $8(19,5)$ & $63(58,3)$ & $31(50,8)$ & \\
\hline \multicolumn{7}{|l|}{ Colesterol HDL-c } \\
\hline Aceitável & $58(92,1)$ & $86(82,7)$ & $24(58,5)$ & $90(83,3)$ & $38(62,3)$ & $<0,001$ \\
\hline Limítrofe + baixo & $5(7,9)$ & $18(17,3)$ & $17(41,5)$ & $18(16,7)$ & $23(37,7)$ & \\
\hline \multicolumn{7}{|l|}{ LDL-c } \\
\hline Aceitável & $24(38,1)$ & $23(22,1)$ & $33(80,5)$ & $39(36,1)$ & $17(27,9)$ & $<0,001$ \\
\hline Limítrofe + aumentado & $39(61,9)$ & $81(77,9)$ & $8(19,5)$ & $69(63,9)$ & $44(72,1)$ & \\
\hline \multicolumn{7}{|l|}{ Glicose } \\
\hline Normal & $45(71,4)$ & $72(69,2)$ & $41(100,0)$ & $67(62,0)$ & $57(93,4)$ & $<0,001$ \\
\hline Alterada & $18(28,6)$ & $32(30,8)$ & $0(0,0)$ & $41(38,0)$ & $4(6,6)$ & \\
\hline
\end{tabular}

Os maiores índices de sobrepeso e obesidade $132,8 \%$ e $28,8 \%)$, triglicerídeos elevados $(37,7 \%$ e $33,7 \%)$ e LDL-c elevados (72,1\% e 77,9\%) foram observados nas escolas 5 e 2, respectivamente, bem como o colesterol total obteve maiores alterações na escola $2(68,3 \%)$ e na escola $1(61,9 \%)$, e o HDL-c obteve maior predomínio na escola $3(41,5 \%)$ e na escola $5(37,7 \%)$. O perfil glicêmico apresentou níveis aumentados principalmente na escola $4(38,0 \%)$, seguido da escola $2(30,8 \%)$.

\section{DISCUS5Ão}

No presente estudo, 59,7\% dos escolares apresentaram valores alterados de LDL-C, 52,4\% alteração nas taxas de CT, e 30,5\% indicaram glicose alterada. Estudo transversal em Vitória-ES, com 511 crianças com idades entre seis e nove anos, verificou taxas menores de CT elevado $(32,7 \%)$ e de $\operatorname{LDL}-\mathrm{c}(27,0 \%)^{18}$. Outro estudo, realizado em Amargosa-BA, com 1.139 escolares de seis a 18 anos de idade, verificou prevalência de CT, triglicerídeos e LDL-c elevados em 23,1\%, $12,4 \%$ e $4,5 \%$ dos sujeitos respectivamente, sendo os resultados inferiores aos relatados em nosso estudo. 0 mesmo estudo também identificou valores mais expressivos de HDL-c baixo em $41,5 \%$ da amostra. ${ }^{19}$

Valores menores que o do presente estudo também foi verificado para CT, LDL-c e triglicerídeos em estudo de âmbito nacional que avaliou 38.069 adolescentes de 12 a 17 anos, em que se observou alteração em 44,3\%, $23,0 \%$ e $21,8 \%$ dos participantes, respectivamente. HDL-c baixo foi observado em $46,8 \%$ dos adolescentes, resultado este mais alto que o evidenciado em nosso estudo. ${ }^{20}$ Já, estudo realizado com crianças e adolescentes da Coréia, com idades entre 10 a 18 anos, verificou níveis alterados de HDL-c em 7,1\%, LDL-c em $4,7 \%$ e triglicerídeos em $10,1 \% .{ }^{21}$

Beck et al. ${ }^{22}$ em estudo transversal com 660 escolares de 14 a 19 anos de Três de Maio-RS, evidenciou alteração de CT e HDL-c em 20,3\% e 25,9\%, respectivamente. Foi observado também que $0,9 \%$ da amostra apresentaram hiperglicemia, diferente de nosso estudo, que verificou glicose alterada em 30,5\% da amostra. Estudo transversal realizado nos Estados Unidos, com 1235 adolescentes de 12 a 19 anos de idade, evidenciou entre os meninos taxas alteradas de 4,3\%, 2,0\%, $18,1 \%$, para triglicerídeos, LDL-c e HDL-c, respectivamente; entre as meninas, observou-se alteração em $5,4 \%$ para triglicerídeos, 3,8\% para LDL-c e $12,2 \%$ para HDL-c. ${ }^{23}$ Outro estudo realizado em Recife-PE verificou, em uma amostra de 470 adolescentes de 10 a 14 anos, níveis limítrofes e alterados de CT, LDL-c e triglicerídeos em $36,2 \%, 14,5 \%$ e $35,3 \%$ respectivamente, e HDL-c alterado em $56 \%$ dos escolares, tendo ambos os estudos evidenciado níveis menores de alteração quando comparado ao presente estudo. ${ }^{14}$

Quanto ao perfil nutricional, nosso estudo demonstrou que $27,0 \%$ da amostra apresentaram sobrepeso e obesidade. Já, outros estudos no Brasil verificaram valores mais preocupantes. Almeida et al. ${ }^{18}$ em estudo na cidade de Vitória-ES, verificou excesso de peso em $38,5 \%$ das crianças avaliadas, sendo que $13,9 \%$ apresentaram sobrepeso e $24,6 \%$ obesidade. Concomitantemente, Lima, Romaldini e Romaldini ${ }^{24}$ em seu estudo com crianças e adolescentes com idades entre cinco e 17 anos, no município de Santa Rita do Sapucaí-MG, observaram que $37,2 \%$ dos escolares apresentaram excesso de peso.

Estudo realizado em escolas da rede privada de ensino da cidade de Divinópolis-MG, com crianças de oito a 10 anos, evidenciou que $33,2 \%$ destas apresentaram sobrepeso/obesidade, tendo o excesso de peso se associado ao sexo e ao peso da mãe. ${ }^{25}$ Já, em Chapada-RS, estudo com crianças de seis a 10 anos de idade, evidenciou que $30 \%$ dos escolares demonstraram excesso de peso, sendo a maior prevalência na zona rural. ${ }^{26}$ Do mesmo modo, Barros et al., ${ }^{27}$ em estudo com adolescentes com média de idade de 12 anos de Carmo-RJ, observou prevalência de sobrepeso/obesidade de $28,9 \%$.

Valores menores aos encontrados em nossa pesquisa também são observados em outros estudos. Alcântara Neto et al. ${ }^{13}$ verificaram ocorrência de sobrepeso e obesidade em 12,4\% em umas amostra de 937 
crianças e adolescentes com idades entre sete e 14 anos, na cidade de Salvador-BA. Já, Souza e Farias ${ }^{28}$ em estudo transversal realizado em Rio Branco-AC, ao avaliar 706 escolares de oito a 14 anos de escolas públicas, verificou prevalência de excesso de peso em 17,6\%.

Em outros países, podem-se observar valores mais expressivos de sobrepeso e obesidade. Sobrero et al. ${ }^{8}$ em estudo com adolescentes de sete províncias da Argentina, com idades entre 10 e 19 anos, verificaram $40,4 \%$ dos indivíduos com sobrepeso ou obesidade. $\mathrm{Na}$ cidade de Beja, Portugal, foram avaliadas crianças e adolescentes com idades entre nove e 18 anos, e observou-se que cerca de $14,4 \%$ dos escolares apresentavam obesidade e $16,7 \%$ sobrepeso, totalizando $31,0 \%$ dos escolares com excesso de peso. ${ }^{29}$

Outros estudos evidenciaram valores menores aos encontrados em nossa pesquisa. Estudo transversal de Boukthir et al. ${ }^{30}$ demonstrou, em uma amostra de 1335 sujeitos em idade escolar de seis a 12 anos de Tunis, Tunísia, prevalência de sobrepeso e obesidade de $19,7 \%$ e $5,7 \%$, respectivamente, totalizando $25,4 \%$ dos escolares com excesso de peso. Em estudo de Pangani et al. ${ }^{31}$ em Dar es Salaam, na Tanzânia, com 1781 crianças e adolescentes com idades entre oito e 13 anos, verificou prevalência de sobrepeso e obesidade de $15,9 \%$ e $6,7 \%$, respectivamente, em um total de $22,6 \%$ com excesso de peso. 0 mesmo estudo também mostrou que o excesso de peso foi mais prevalente entre as crianças, na faixa etária de oito a 10 anos, quando comparado ao grupo etário dos 11 aos 13 anos.

No nosso estudo, houve diferença significativa entre as escolas em todas as variáveis avaliadas do perfil lipídico e da glicose para ambos os sexos. Do mesmo modo, destaca-se que para o sexo masculino as escolas 2 e 4 apresentaram as maiores alterações, e para o sexo feminino as escolas 2 e 5 . Quanto a localização destas escolas, a escola 5 situa-se distante da área urbana, com muitos aspectos marcantes rurais e com filhos de agricultores auxiliando na lavoura. Diferentemente, a escola 2, mesmo não geograficamente próxima ao centro, situa-se em um distrito municipal com muitas características urbanas, em que o desenvolvimento e crescimento urbano e econômico tem alcançado grandes proporções. Concomitantemente, a escola 4 situa-se em região asfaltada, com comércio e linhas de ônibus em suas proximidades, apresentando também características urbanas. Tais características urbanas podem ter levado ambas as escolas a adotarem algumas características e hábitos de vida da zona urbana, as quais podem ter vindo a influenciar os presentes resultados desfavoráveis a essas escolas, demonstrando que a localização da escola pode interferir no perfil lipídico e glicêmico de escolares.

Estudo realizado no mesmo município de nossa pesquisa verificou as possíveis diferenças na aptidão física relacionada à saúde de crianças e adolescentes dos diferentes hemisférios da zona rural de Santa Cruz do Sul - RS, e concomitantemente ao nosso estudo, houve diferença significativa entre as escolas, em que as escolas mais afastadas geograficamente da zona urbana apresentaram índices significativamente superiores nas variáveis força/resistência abdominal e aptidão cardiorrespiratória em relação às outras escolas, para ambos os sexos. ${ }^{32}$ Outro estudo, no município, também encontrou diferença significativa entre as escolas da zona rural, porém em relação à aptidão física relacionada ao desempenho motor, em que se observaram diferenças significativas quanto à força de membros inferiores, velocidade e agilidade para o sexo masculino e força de membros superiores, velocidade e agilidade, no sexo feminino. Porém, não se observou que escolas com evidentes características urbanas foram as que demonstraram os piores resultados quanto ao desempenho nos testes avaliados. ${ }^{33}$

Como ponto forte de nosso estudo, pode-se mencionar o número elevado de crianças e adolescentes, evidenciando uma amostra representativa de escolares do município. Outro ponto a destacar é o comparativo entre as escolas com diferentes características de localização, sendo que poucos estudos consideram as distinções existentes entre as escolas. Por outro lado, o estudo apresenta limitações, como a natureza transversal da pesquisa, a qual não possibilita a verificação de relação de causa e efeito.

\section{CONSIDERACQÕES FINAIS}

Houve elevado percentual de alterações no perfil de risco cardiovascular dos escolares (nutricional, lipídico e glicêmico). Quanto ao comparativo entre escolas, o estudo apontou diferença significativa entre as escolas em todas as variáveis avaliadas do perfil lipídico e para a glicose em ambos os sexos, demonstrando que a localização da escola pode interferir no perfil lipídico e glicêmico de escolares.

Esse estudo determinou a distribuição do índice nutricional, lipídico e glicêmico em uma amostra de escolares da zona rural de Santa Cruz do Sul (RS), e apesar dos dados relatados nesse estudo serem atribuídos a apenas uma localidade, o trabalho é válido pela contribuição à área científica e pelos dados adquiridos referentes à parte Sul do Brasil. Assim, medidas que visem à verificação do perfil de risco cardiovascular na infância e adolescência podem ter um importante impacto sobre as doenças cardiovasculares no futuro desses escolares.

\section{REFERÊNCIAS}

1. De Onis M, Blossner M, Borghi E. Global prevalence and trends of overweight and obesity among preschool children. Am J Clin Nutr 2010;9(2):1257-64. doi: 10.3945/ ajcn. 2010.29786.

2. Pereira-Lancha LO, Campos-Ferraz PL, Lancha $A H$ JR. Obesity: considerations about etiology, metabolism, and the use of experimental models. Diabetes Metab Syndr Obes 2012;5:75-87. doi: 10.2147/DMSO.S25026

3. Martínez-Gómez D1, Welk GJ, Calle ME, Marcos A, Veiga OL. Preliminary evidence of physical activity levels measured by accelerometer in Spanish adolescents: the AFINOS Study. Nutr Hosp 2009;24(2):226-32.

4. Lewis GF. Devastating metabolic consequences of a life of plenty: focus on the dyslipidemia of overnutrition. Clin Invest Med 2013;36(5):E242-E247.

5. Abrams P, Levitt Katz LE. Metabolic effects of obesity causing disease in childhood. Curr Opin Endocrinol Diabetes Obes 2011;18(1):23-7. doi: 10.1097/MED.0b013e3283424b37.

6. Jodkowska M, Oblacińska A, Tabak I, Radiukiewicz K. 
The role of physical education teacher in the active care of pupils with overweight and obesity. Med Wieku Rozwoj 2010;14(2):197-206.

7. Di Renzo L, Tyndall E, Gualtieri P, Carboni C, Valente R, Ciani AS, Tonini MG, De Lorenzo A. Association of body composition and eating behavior in the normal weight obese syndrome. Eat Weight Disord 2016;21(1):99-106. doi: 10.1007/s40519-015-0215-y.

8. Figueroa Sobrero A, Evangelista $P$, Kovalskys I, Digón $P$, López S, Scaiola E, Perez N, Dieuzeide G, Walz F, Mazza C. Cardio-metabolic risk factors in Argentine children. A comparative study. Diabetes Metab Syndr 2016;(1 Suppl 1):S103-9. doi: 10.1016/j.dsx.2015.10.003

9. Magalhães EIS, Sant'Ana LFR, Priore SE, Franceschini SCC. Waist circumference, waist/height ratio, and neck circumference as parameters of central obesity assessment in children. Rev Paul Pediatr 2014;32(3):273-281. doi: 10.1590/0103-0582201432320

10. Bergmann GG, Gaya A, Halpern R, Bergmann MLA, Rech RR, Constanzi CB, Alli LR. Body mass index to the cardiovascular disease risk screening in infancy. Arq Bras Endocrinol Metab 2011;55(2):114-120. doi: 10.1590/S000427302011000200003.

11. Mirand JM, Ornelas EM, Wichi RB. Childhood obesity and cardiovascular risk factors. ConScientiae Saúde 2011(10):175-180.

12. Franca EF, Alves JGB. Dyslipidemia Among Adolescents and Children from Pernambuco. Arq Bras Cardiol 2006;87(6):722-727.

13. De Alcântara Neto OD, Silva $R$ de C, Assis AM, Pinto E de J. Factors associated with dyslipidemia in children and adolescents enrolled in public schools of Salvador, Bahia. Rev Bras Epidemiol 2012;15(2):335-45.

14. Pereira PB, Arruda IKG, Cavalcanti AMTS, Diniz AS. Lipid Profile of Schoolchildren from Recife, PE. Arq. Bras. Cardiol 2010;95(5):606-613. doi: 10.1590/S0066$782 \times 2010005000136$

15. NHLBI. National Heart, Lung, and Blood Institute. Expert panel on integrated guidelines for cardiovascular health and risk reduction in children and adolescents. Bethesda: National Heart, Lung, and Blood Institute, 2012.

16. ADA. American Diabetes Association. Diagnosis and classification of diabetes mellitus. Diabetes Care 2015;38(1):s8-s16. doi: 10.2337/dc10-S062

17. $\mathrm{CDC} / \mathrm{NCHS}$. Centers for Disease Control and Prevention/ National Center for Health Statistics. CDC Growth Charts: United States. 2000. Disponível em: http://www.cdc.gov/nchs/ data/series/sr 11/sr11 246.pdf. Acesso em: 15 set. 2016.

18. Almeida PCD, Silva JP, Pinasco GC, Hegner CC, Mattos DC, Potratz MO, Bravin LS, SilvaVR, Lamounie JL. Lipid profile in school children in Vitória - Brazil. Hum Growth Dev 2016;26(1):61-66. doi: 10.7322/jhgd.110989

19. Quadros TMB, Gordia AP, Silva LR, Silva DAS, Mota J. Inquérito epidemiológico em escolares: determinantes e prevalência de fatores de risco cardiovascular. Cad Saúde Pública 2016;32(2):e00181514. doi: 10.1590/0102-311x00181514

20. Neto Faria JR, Bento VFR, Baena CP, Olandoski M, Gonçalves LGO, Abreu GA, Kuschnir MCC, Bloch KV. ERICA: prevalência de dislipidemia em adolescentes brasileiros. Rev Saude Publica 2016;50(Suppl 1):10s. doi: 10.1590/S01518

\subsection{3}

21. Yang S, Hwang JS, Park HK, Lee HS, Kim HS, Kim EY, Lim JS. Serum Lipid Concentrations, Prevalence of Dyslipidemia, and Percentage Eligible for Pharmacological Treatment of Korean Children and Adolescents; Data from the Korea National Health and Nutrition Examination Survey IV (20072009). PLoS One 2012;7(12):e49253. doi: 10.1371/journal. pone.0049253

22. Beck CC, Lopes AS, Giuliano ICB, Borgatto AF. Fatores de risco cardiovascular em adolescentes de município do sul do Brasil: prevalência e associações com variáveis sociodemográficas. Rev Bras Epidemiol 2011;14(1):36-49. doi: 10.1590/S1415-790×2011000100004

23. Leblanc AG, Janssen I. Dose-response relationship between physical activity and dyslipidemia in youth. Can J Cardiol 2010;26(6):e201-e205.

24. Lima MCC, Romaldini CC, Romaldini JH. Frequency of obesity and related risk factors among school children and adolescents in a low-income community. A crosssectional study. Sao Paulo Med J 2015;133(2):125-30. doi: 10.1590/1516-3180.2014.8960412

25. Ferreira SD, Carballo FP, Sousa FF, Silva DMR. Prevalência e fatores associados ao sobrepeso/obesidade e à hipertensão arterial sistêmica em crianças da rede privada de ensino de Divinópolis/MG. Cad Saúde Colet 2015;23(3):289-97. doi: 10.1590/1414-462X201400060082

26. Polla SF, Scherer F. Perfil alimentar e nutricional de escolares da rede municipal de ensino de um município do interior do Rio Grande do Sul. Cad Saúde Colet 2011;19(1):111-6.

27. Barros MS, Fonseca VM, Meio MDBB, Chaves CR. Excesso de peso entre adolescentes em zona rural e a alimentação escolar oferecida. Cad Saúde Colet 2013;21(2):201-08. doi: 10.1590/S1414-462X2013000200016

28. Souza OF, Farias ES. Underweight and overweight in school children from Rio Branco, Acre State, Brazil. Rev Bras Cresc Des Hum 2011;21(3):878-82.

29. Lourenço J. Prevalência da obesidade em meio escolar, estudo realizado ao segundo e terceiro ciclo de escolaridade numa escola na cidade de Beja. Rev Educ Motr Inv 2015;5:61-8.

30. Boukthir S, Essaddam L, Mazigh Mrad S, Ben Hassine L, Gannouni S, Nessib F, Bouaziz A, Brini I, Sammoud A, Bouyahia $\mathrm{O}$, Zouari B. Prevalence and risk factors of overweight and obesity in elementary schoolchildren in the metropolitan region of Tunis, Tunisia. Tunis Med 2011;89(1):50-4.

31. Pangani IN, Kiplamai FK, Kamau JW, Onywera VO. Prevalence of Overweight and Obesity among Primary School Children Aged 8-13 Years in Dar es Salaam City, Tanzania. Adv Prev Med 2016;2016:1345017. doi: 10.1155/2016/1345017

32. Henkes CM, Borfe L, Muradás R, Tornquist L, Burgos MS. Aptidão física relacionada à saúde de escolares: estudo comparativo dos hemisférios Norte - Sul - Leste - Oeste da zona rural de Santa Cruz do Sul - RS. Cinergis 2013;14(4):206-209.

33. Jochims S, Zeni AE, Nunes HMB, Borfe L, Burgos MS. Aptidão física relacionada ao desempenho motor de escolares: estudo comparativo dos hemisférios Norte - Sul - Leste - Oeste, da zona rural de Santa Cruz do Sul - RS. Cinergis 2013;14(2):143-7.

Como citar: BARBIAN, Cláudia Daniela et al. Comparação do perfil nutricional, lipídico e glicêmico de crianças e adolescentes de diferentes hemisférios da zona rural de Santa Cruz do Sul-RS. Cinergis, Santa Cruz do Sul, v. 18, n. 2, mar. 2017. ISSN 21774005. Disponível em: <https://online.unisc.br/seer/index.php/cinergis/article/view/9018>. Acesso em: 29 mar. 2017. doi:http:// dx.doi.org/10.17058/cinergis.v18i2.9018. 